\title{
FARAD: A storage system for random real-time data
}

\author{
Takashi TOTSUKA
}

\author{
SONY Corp., 6-7-35 Kita-Shinagawa, Shinagawa, Tokyo 141, Japan
}

\begin{abstract}
Many multimedia applications demand real-time and high throughput data retrieval from a storage subsystem. Very often, the access pattern of such retrieval has random nature. This is due to real-time playback of video clips that are physically separated on the disk platter (non-linear editing), or due to serving many independent clients simultaneously (VOD; video on demand).

In this paper, we describe a new real-time disk management algorithm that can significantly reduce the overhead incurred by random access. While existing algorithms treat the rotational delay as uncontrollable, our algorithm controls both seek and rotational delay by proper scheduling and data placement so as to reduce overhead in a random data access environment.
\end{abstract}

\section{Keywords:}

Head scheduling, SCAN algorithm, real-time file system, block allocation.

\section{INTRODUCTION}

High throughput and real-time response ${ }^{1}$ are fundamental requirements for a storage system for multimedia data such as movie and audio. Moreover, important multimedia applications demand those in a random data access environment, making the design of a storage system yet harder.

For example, random access is essential in VOD in order to serve concurrently, requests from many clients for they access different images from different movies at different speeds. It is also essential in non-linear editing in which fragments of video clips are stored in physically isolated location on disks and are collected and output at realtime when the play button is pressed. Unfortunately, disk performance degrades - sometimes dramatically - due to the overhead (i.e., seek time and rotational delay) incurred by random access.

Of course, the overhead can be kept relatively small by choosing a large disk $\mathrm{I} / \mathrm{O}$ size. However, such large $\mathrm{I} / \mathrm{O}$ size is often impractical due to increased latency and/or fragmentation. Furthermore, the RAID configuration requires that a logical block of movie and/or audio must be scattered

\footnotetext{
${ }^{1}$ Upper bound of the worst case is given.
}

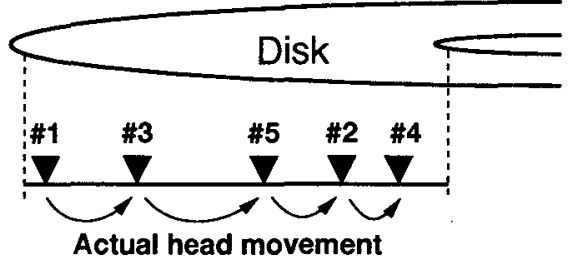

Figure 1: SCAN scheduling.

across several disks. Consequently, the physical I/O size cannot be large unless the logical block can be chosen arbitrary. For image data such as MPEG2 and CCIR601 ${ }^{2}$ and for audio such as AES/EBU ${ }^{3}$, disk I/O size is around $10 \sim$ $500 \mathrm{~KB}$. For a recent high speed hard disk drive (HDD), this amount of data can be retrieved in about several milliseconds to a couple of tens of milliseconds. Since the sum of worst case seek and rotational delay is about $30 \mathrm{~ms}$, without a proper disk access management, most of the total I/O time would be spent by the overhead.

Furthermore, recent increase in media density provides yet shorter data read/write time. Since the increase of the density is much faster than mechanical performance improvements (i.e., seek time and rotational delay), the above mentioned overhead is becoming a more serious problem.

Our disk management algorithm, called FARAD (FAst Random Access Disk management), addresses this problem and can significantly reduce the worst case overhead. Unlike existing algorithms, the FARAD algorithm can reduce the rotational delay under the worst case condition to nearly zero, providing much lower upper bound of the $\mathrm{I} / \mathrm{O}$ time. Efficient real-time random access that is necessary for many multimedia applications is made possible by this algorithm.

The rest of the paper is organized as follows. In section 2, we review related works. The algorithm is described in section 3 and the results of our experiments are shown in section 4.

\footnotetext{
${ }^{2}$ Popular uncompressed image format used in professional video production and broadcasting

${ }^{3}$ Uncompressed.audio format commonly used in professional studios.
} 


\section{PREVIOUS WORKS}

Disk management has a long history. Since seek time was the major component of the overhead, early studies focused on reduction of the seek time. There has been two approaches to address the problem - head scheduling and file allocation.

Head scheduling is a technique to reduce the total distance of head movements by reordering the $\mathrm{I} / \mathrm{O}$ requests. Many algorithms were proposed [3]. The SCAN algorithm, for example, sorts $\mathrm{V} / \mathrm{O}$ requests radially and serves them in the sorted order. By this sorted service, the SCAN can reduce zig-zag head movement that would occur if requests were served in the order they arrive $(\# 1, \# 2, \ldots)$. See fig. 1. Seek time can be reduced by file allocation as well. The fast file system (FFS) of the Berkeley Unix and recent LFS of Sprite are examples. They exploit locality of data by placing relevant objects in the neighborhood.

These studies, however, attempted to improve the average performance, not the worst case performance (real-time performance) of the storage system. Studies of real-time file system are relatively new. A review is found in [7].

A framework of real-time file system was done by Anderson et al[1]. In [1], disk management was discussed as an optimization problem of $\mathrm{I} / \mathrm{O}$ size and buffer requirement under real-time constraints. In their study, the worst case seek time and rotational delay (one revolution) were added to each I/O to simplify their analysis. Rangan et al [10] discussed conditions of segment length and length between segments to satisfy the real-time requirements. In their paper also, the worst case seek and rotational delay was added to each $\mathrm{I} / \mathrm{O}$. As in these papers, performance analysis becomes easier by regarding the overhead as a constant value and choosing the sum of the worst case seek time and rotational delay as the constant. Although this is a safe approach, the overhead estimated in this way is a loose upper bound and the resulting performance estimate is not useful in practice.

Attempts were made to give tighter upper bound by analyzing the scheduled head movement more in detail. Preseeking sweep by Gemmell et al[5][6], Grouped sweeping scheme by Chen et al [2], SCAN-EDF by Reddy and Wyllie[11] are examples.

All these algorithms are real-time extensions to the SCAN algorithm. In order to guarantee the upper bound of $\mathrm{I} / \mathrm{O}$ time, they impose restrictions on $\mathrm{I} / \mathrm{O}$ request queuing. In [6] [2] [12], the order of service to clients is restricted. In [11], the depth of reorder within a stream is restricted. Since the performance analysis was made under optimized head movement by SCAN, these studies give tighter and better upper bound of the seek time.

These algorithms, however, didn't either control or give precise analysis of the rotational delay. [5] treats rotational

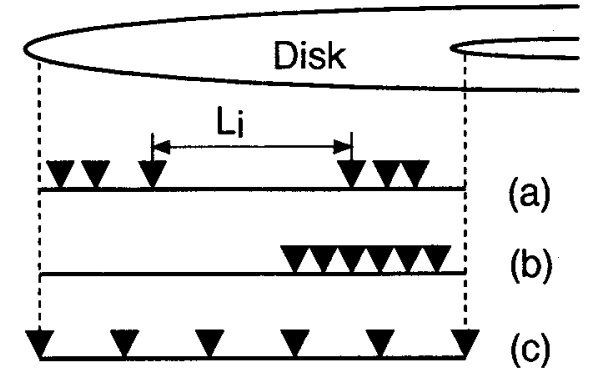

Figure 2: Disk access patterns.

delay as uncontrollable and always chooses the worst case delay (one revolution). Although this is safe, the resulting worst case performance is unnecessarily lower than the average.

Some HDDs have the zero-latency access mechanism. With this mechanism, the HDD starts reading the data immediately after the head reaches the target track even if the head is at the middle of the data to be read. The missed portion of the data is read later on the next revolution. When the $\mathrm{I} / \mathrm{O}$ size is almost one track, the mechanism can nearly eliminate the rotational delay as in the assumption in [11]. However, this mechanism is useful only when a track is nearly filled with useful data. This is not the case if the $\mathrm{I} / \mathrm{O}$ size is small or an I/O spans across multiple tracks (e.g., fills a half of the first track and another half of the second track). Furthermore, commercially available HDDs don't support this mechanism or even if it is supported, its implementation is very limited.

Some papers claim that the rotational delay is negligible [2]. However, this is no longer true with recent HDDs with small seek time. With the SCAN scheduling, the worst case seek time can be easily kept below about $6 \mathrm{~ms}^{4}$. This is smaller than the revolution period, which is $8.3 \mathrm{~ms}$ for a $7200 \mathrm{rpm}$ HDD. Required power and generated heat due to resistance of air make it difficult to increase the rotational speed dramatically.

Hence, rotational delay is the dominant factor and its importance will not decrease in the future.

$\mathrm{Ng}$ et al [9] also pointed out the importance of rotational delay reduction. In his paper, multiple copies of data are used to reduce rotational delay during read. Kajitani[8] also proposed a use of mirrored disk for performance improvement. However, use of multiple copies is prohibitive in multimedia applications since the size of data is typically very large. 


\section{OPTIMIZING SEEK AND ROTATIONAL DELAY}

\subsection{Seek time analysis}

As shown in fig. 2 , there are many $1 / O$ request patterns that a single scan accesses. In order to guarantee real-time response, we must find the worst case request pattern that maximizes the sum of $\mathrm{H} / \mathrm{O}$ overhead within a scan.

First, we ignore for the moment rotational delay and assume that the I/O overhead $T_{\text {delay }}$ is determined solely by seek time. Let $T_{\text {total }}$ be the sum of the overhead. Clearly, $T_{\text {total }}=\sum T_{\text {delay }}$. Let a series $L=\left\{L_{1}, L_{2}, L_{3}, \ldots\right\}$ be the distances in cylinders between adjacent $1 / O$ requests. Our job is to find a particular $L$ that maximizes $\mathcal{T}_{\text {total }}$.

Under the above assumption, $T_{\text {total }}$ is given by equ. 1 .

$$
T_{\text {total }}=\sum_{i=1}^{N_{i o}-1} T_{s e e k}\left(L_{i}\right)
$$

where $T_{\text {seek }}(n)$ is the seek time to travel $n$ cylinders and $N_{i o}$ is the number of $\mathrm{T} /$ Os serviced in one scan.

Since I/O requests may be made to any cylinders, the total seek distance in a scan is as long as the total number of cylinders $N_{c y l}{ }^{5}$. Thus, we should find $L$ that maximizes equ. 1 under the constraint in equ. 2 .

$$
\sum_{i=1}^{N_{i 0}-1} L_{i}=N_{c y i}
$$

For a concave down function such as seek profile, it can be shown that the sum of the overhead $T_{\text {total }}$ is maximum when all the $\mathrm{I} / \mathrm{O}$ requests are evenly distributed as in fig. 2(c). The proof is shown in appendix A. When $T_{\text {total }}$ is maximum, the average $T_{\text {delay }}$ is given as

$$
\text { Avg. } T_{\text {delay }}=T_{\text {seek }}\left(\frac{N_{c y l}}{N_{i o}-1}\right)
$$

where $L_{a v g}$ is the average seek distance given by $L_{a v g}=$ $\frac{N_{c y l}}{N_{\text {io }}-1}$.

Of course, there are many request patterns in which $T_{\text {delay }}$ for a certain $1 / O$ is much bigger than the above average (equ. 3). However, in such cases, the overhead for other VOS are much smaller than that and the average overhead of the entire scan never exceeds equ. 3 . As the request pattern distribution becomes more even, the total $\mathrm{I} / \mathrm{O}$ time gets closer to its worst value. This property holds for any $T_{\text {delay }}$ function as long as it is concave down. We will use this property when we analyze rotational delay.

\footnotetext{
${ }^{4}$ With 10 or more I/Os per a scan.

${ }^{5}$ Precisely speaking, the distance is shorter by the amount occupied by the data to be accessed data. But this is usually negligible compared to the $N_{\text {cyl }}$ which is $2000 \sim 4000$.
}

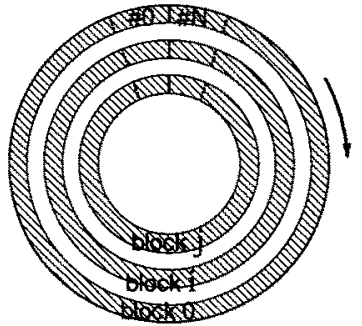

(a)

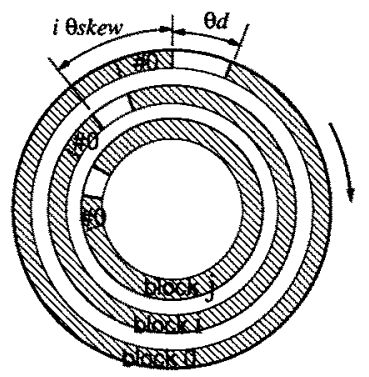

(b)
Figure 4: Physical layout of of lgical blocks. (a) Aligned, (b) skewed.

\subsection{Real delay with SCAN}

In the previous section, we ignored rotational delay. In reality, we have to add a period of one revolution $\left(T_{\text {rot }}\right)$ or the worst case since we don't control the rotational delay so far. Thus, the overhead $T_{\text {delay }}$ of SCAN is equal to the seek profile shifted by the amount of one revolution (fig. 3(a)). This function also is concave down, and hence, the worst case arises when all the $\mathrm{VO}$ requests are evenly distributed. The worst case average overhead is given by reading the chart at the average seek distance $L_{\text {avg }}$.

$$
\text { Avg. } T_{\text {delay }}=T_{\text {seek }}\left(\frac{N_{c y l}}{N_{i o}-1}\right)+T_{\text {rot }}
$$

\subsection{Controlling rotational delay}

We now show that a proper placement of logical blocks can nearly eliminate rotational delay from the above worst case average I/O overhead. To begin with, let's assume that (1) all the logical blocks starts at the same physical sector (same orientation viewed from the center) and that (2) the size of a logical block is multiple of a track as shown in fig. 4(a). In this particular format, when an access is finished for a logical block, the start of the next logical block is already under the head. Thus, even for a short movement to a neighbor (e.g., from block \#0 to \#1), we have to wait for another revolution. Hence, for a short seek $T_{\text {delay }}=T_{\text {rot }}$ and $T_{\text {delay }}$ remains unchanged $\left(T_{\text {rot }}\right)$ as long as $T_{\text {seek }}<T_{\text {rot }}$. When $T_{\text {seek }}$ is equal to $T_{\text {rot }}$, rotational delay becomes zero and for any further seek, $T_{\text {delay }}$ becomes $2 T_{\text {rot }}$. See fig. 3(b).

In the previous analysis of SCAN, the actual $T_{\text {detay }}$ was unknown. Only its upperbound can be plotted (fig. 3(b)). However, by specifying the placement of logical blocks, $T_{\text {delay }}$ becomes a deterministic value. In other words, the rotational delay is now a controllable value.

As the second step, we consider giving angular skew to the start of logical blocks. Let $\theta_{s k e w}$ be skew angle in degree between the start of two adjacent blocks, and $\theta_{\text {gap }}$ be the gap 


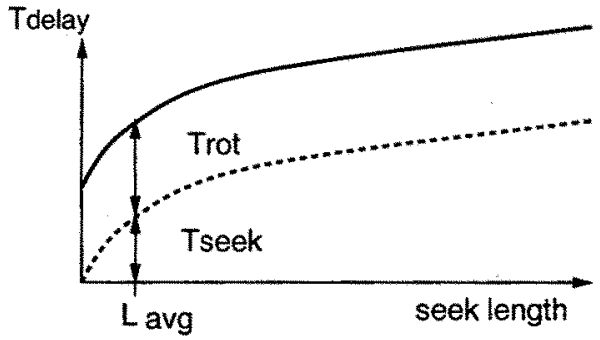

(a)

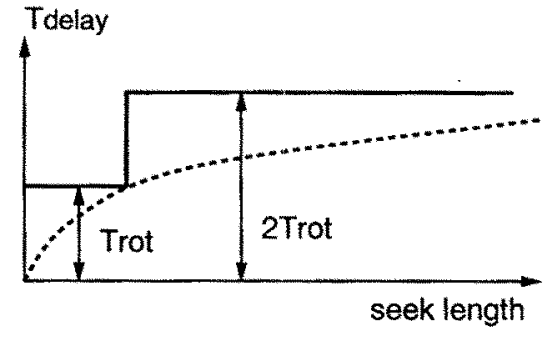

(b)

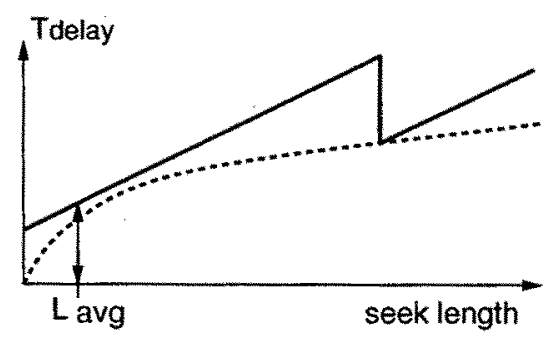

(c)

Figure 3: Disk access overhead. (a) Without allocation constraints, (b) aligned layout, (c) skewed layout.

between the start and the end of a logical block. Fig. 4(b) shows an example of this skewed allocation scheme.

Suppose an access to a logical block is just finished and the head is about to seek to another block which is $L$ cylinder away. Then the angle to the start of that block is given by $L \theta_{\text {skew }}+\theta_{\text {gap }}$. If the head seek completes before the disk rotates this amount, then the delay is given by dividing this angle by the rotation speed. If the seek does not complete, the head has to wait for one revolution or $T_{\text {rot }}$ is added to the delay. If, on the other hand, the head can catch the start of the destination block one revolution earlier, $T_{\text {rot }}$ is subtracted from the delay. As a result, $T_{\text {delay }}$ is a saw-tooth function as shown in fig. 3(c).

In equation, $T_{\text {delay }}$ for this skewed allocation scheme is given as

$$
T_{\text {delay }}(L)=\left(\frac{L \theta_{s k e w}+\theta_{\text {gap }}}{360}+m\right) T_{\text {rot }}
$$

where $L$ is the seek distance. $m$ is the smallest integer that satisfies the condition $T_{\text {delay }}>T_{\text {seek }}$.

Note that the slope and the value at zero seek of $T_{\text {delay }}$ are determined by $\theta_{\text {skew }}$ and $\theta_{g a p}$, respectively. Since $\theta_{s k e w}$ is a design parameter and $\theta_{g a p}$ is indirectly determined by logical block size which again is a system design parameter, the entire shape of $T_{\text {delay }}$ is controllable. As a special case, when $\theta_{\text {skew }}=\theta_{\text {gap }}=0$, the delay function shown in fig. $3($ b) is obtained.

Although fig. 4(b) shows a format example of one track, the principle holds for larger block sizes that requires multiple tracks and/or multiple cylinders.

Since $T_{\text {delay }}$ is no longer a concave down function, analytically exact worst case analysis for the skewed allocation scheme is difficult. However, a good approximation can be easily obtained. For overhead functions such as fig. 3(b)and(c), we can easly find a concave down function that bounds the delay. Examples are shown in fig. 5 with solid lines. Once such concave down function is obtained, the rest of the argument is similar to previous sections. The worst case of the sum of the overhead ( $T_{\text {total }}$ ) is given when $\mathrm{I} O \mathrm{O}$ requests are evenly distributed. Thus, the average $T_{\text {delay }}$ for the
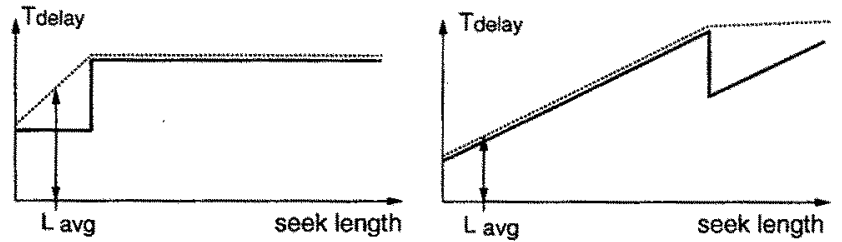

Figure 5: Bounding the delay functions (solid line) by concave down functions (dotted line).

worst case is again simply given by $T_{\text {delay }}\left(L_{\text {avg }}\right)$. Since the bounding function is equal to the actual $T_{\text {delay }}$ near $L_{a v g}$ in our skewed allocation, this approximation gives a good bound.

The above analysis means that, by choosing the logical block format such that $T_{\text {delay }}$ is small near $L_{\text {avg }}$, the worst case overhead can be significantly reduced compared to the existing algorithms that use SCAN alone. The worst case average $T_{\text {delay }}$ is minimum when it is a tangent line of the seek profile. In this case, the rotational delay is totally eliminated as far as the worst case performance analysis is concerned. Of course, the average performance is also improved in this scheme as we see in the later section.

\section{EXPERIMENTAL RESULTS}

\subsection{Parameters used}

In our experiments, the following parameters are used.

$$
\begin{array}{ll}
T_{\text {rot }} & : 8.33 \mathrm{~ms} \\
\text { //O size (avg.) } & : 126 \mathrm{~KB}(246.5 \text { sectors }) \\
\theta_{\text {gap }} & : 111.8 \sim 160.2^{\circ} \\
\theta_{\text {skew }} & : 0.65^{\circ} \\
L_{\text {avg }} & : 197.5 \\
N_{\text {io }} & : 10
\end{array}
$$

These parameters are chosen to simulate the following scenario - a 10-bit CCIR601 video clip storage in which each frame is divided and stored on 8 disks under $7+1$ RAID configuration. 


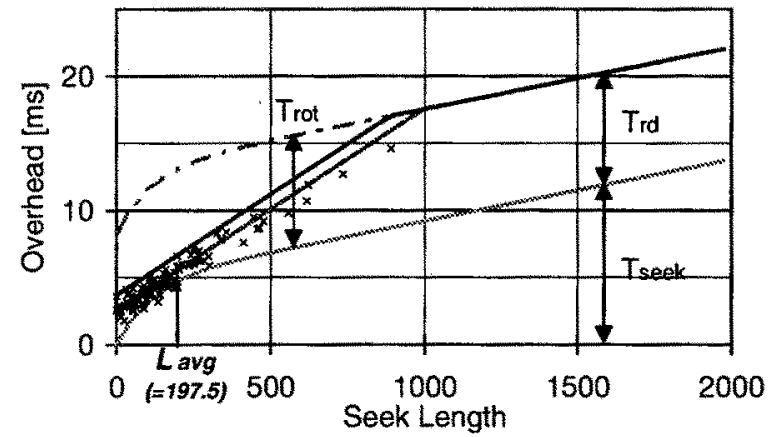

Figure 6: Plot of measured $\mathrm{I} / \mathrm{O}$ overhead. $100 \mathrm{~V} / \mathrm{O}$ s are sampled. Solid lines correspond to the theoretical $T_{\text {delay }}$ for $\theta_{\text {gap }}=111.8$ (lower line) and 160.2 (upper line).

\subsection{Overhead of one $1 / O$}

Fig. 6 shows the plot of actually measured overhead for each disk $\mathrm{I} / \mathrm{O}$. Out of $10^{5}$ randomly generated $\mathrm{V} / \mathrm{Os}$, the overhead of the first 100 I/Os are plotted. The two solid lines correspond to the theoretical $T_{\text {delay }}$ for $\theta_{\text {gap }}=111.8$ and 160.2 , respectively. As shown in the graph, both seek time and rotational delay are well controlled by the proposed skewed allocation scheme. The measured values are similar to the theoretically derived $T_{\text {delay }}$.

Despite the relatively large difference in $\theta_{\text {gap }}$, its actual impact to the performance is minor.

\subsection{Total overhead per one scan}

Fig. 7 shows histograms of the sum of overhead in one scan $\left(T_{\text {total }}\right)$. Each scan consists of 10 V/O requests and $10^{4}$ scans were performed. These $\mathrm{V} / \mathrm{O}$ requests were made to random locations on a disk. For comparison, the sum of the overhead for conventional SCAN and CSCAN (without control of rotational delay) and naive requested order I/O service (without head scheduling) are also shown.

Tab. 1 shows some statistical numbers of this experiment. The first column is the maximum total $1 / O$ overhead derived mathmatically based on analysis. This maximum value may not be observed in a certain experiment. The rest of the columns are actually measured in our experiment.

The figure and the table show the followings.

- All the measured scans are within the upper bound derived theoretically. This verifies the FARAD skewed allocation algorithm.

- The FARAD algorithm exhibits much better wors case performance. The average performance is also better.

- With the FARAD algorithm, the difference of the worst and the average performance is smaller. Oweing to this nice property, system design can be less redundant.

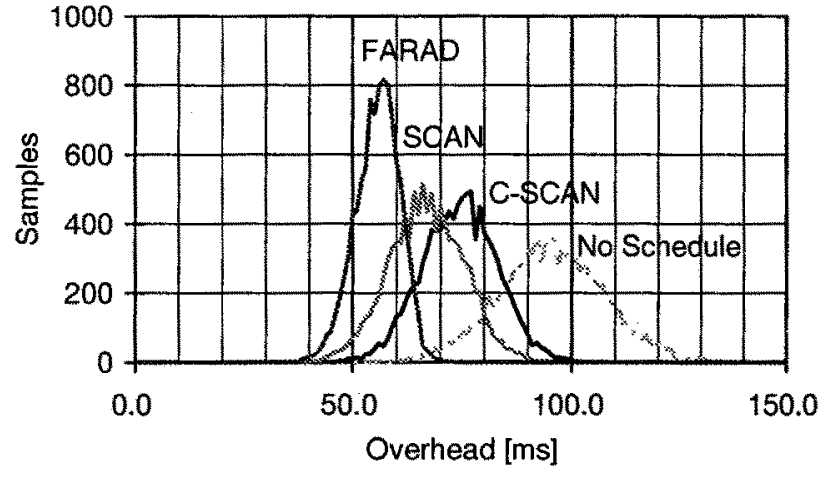

Figure 7: Total I/O Time Histgrams.

\begin{tabular}{|l||c|c|c|c|}
\hline & $\begin{array}{c}\text { Max } \\
\text { (theory) }\end{array}$ & $\begin{array}{c}\text { Max } \\
\text { (observed) }\end{array}$ & Min. & Avg. \\
\hline \hline FARAD & 75.0 & 74.1 & 28.9 & 56.7 \\
\hline SCAN & 130.8 & 95.1 & 27.2 & 66.8 \\
\hline C-SCAN & 141.9 & 105.0 & 34.1 & 74.4 \\
\hline
\end{tabular}

Table 1: Distribution of total UO overhead per scan $\left(T_{\text {total }}\right)$.

- For conventional algorithms, observed maximum overhead is much less (about 70\%) than the theoretically derived maximum. Since a system designer still has to use this theoretically derived maximum for the design, this means that he/she has to prepare for a very rare worst case in the design.

\section{CONCLUSIONS}

A low overhead disk management algorithm for random real-time access is proposed. While existing head scheduling algorithms only reduce seek time, proposed algorithm can reduce both seek time and rotational delay by a judicious combination of a scan based head scheduling and the skewed block allocation scheme. We believe this is the first time that rotational delay is controlled in a clean way without having multiple data copies. The results of some experiments are shown.

Since many multimedia applications require random and real-time data access, our disk management algorithm can provide major server performance improvement in this area.

Although these are beyond the scope of this paper, in real applications, we have to also address subtle issues due to zone bit recording and logical disk model provided by SCSI interface. A RAID support under FARAD scheme must also be developed. We have solved these issues partly by close collaboration with a disk manufacturer and a commercial implementation for professional video post-production system is soon available. 


\section{REFERENCES}

[1] Anderson, D., Osawa, Y., and Govindan, R., "A File System for Continuous Media", ACM Trans. on Computer Systems, vol.10, No.4, pp.311-337, 1992.

[2] Chen, M., Kandlur, D., and Yu, P., "Optimization of the Grouped Sweeping Scheduling (GSS) with Heterogeneous Multimedia Streams", Proc. ACM Multimedia 93, pp.235-242, 1993.

[3] Deitel, H. M., Operating Systems - 2nd ed., AddisonWesley, 1990.

[4] Ellis, G. P., "Disk recording for real time post production applications", IEE Colloquium on 'Recording Media for Television' (Digest No. 1993/239), pp. 5/1-7 of $39,1993$.

[5] Gemmell, J., "Multimedia network file servers : Multi-channel delay sensitive data retrieval", Proc. ACM Multimedia 93, pp.243-250, 1993.

[6] Gemmell, J., Han, J., et. al., "Delay-Sensitive Multimedia on Disks", IEEE Multimedia 1994, pp.56-67, 1994.

[7] Gemmell, J., Vin, H.M., et. al., "Mutimedia Storage Servers: A Tutorial", IEEE Computer, Vol.28, No.5, pp.40-49, 1995.

[8] Kajitani K., "Analysis of Disk Array Management for Digital Video Server", Trans. of the institute of electronics, information and communication engineers, Vol.J77-D-I, No.1, pp.66-76, 1994.

[9] Ng, S. W., "Improving Disk Performance Via Latency Reduction", IEEE Transactions on Computers, Vol.40, No.1, pp.22-30, 1991.

[10] Rangan, V., and Vin, H., "Efficient Storage Techniques for Digital Continuous Multimedia", IEEE Trans. on knowledge and data eng., vol.5, No.4, pp.564-573, 1993.

[11] Reddy, N., Wyllie, J., "Disk scheduling in a multimedia I/O system", Proc. ACM Multimedia 93, pp.225233, 1993.

[12] Yu, P., Chen, M., and Kandlur, D., "Design and Analysis of a Grouped Sweeping Scheme for Multimeda Storage Management", 3rd Int'l Workshop on Network and Operating System Support for Digital Audio and Video, pp.44-55, 1992.

\section{Appendix A}

Claim.

If a function $f(x)$ is concave down ${ }^{6}$ and satisfies the constraint $\sum_{i=1}^{n} x_{i}=X$, then the maximum value of $\sum_{i=1}^{n} f\left(x_{i}\right)$ is obtained when $x_{i}=X / n$.

Base case:

Consider when $n=2$. Let $T(x)$ be $f(x)+f(X-x)$. Then
$T^{\prime}(x)=f^{\prime}(x)-f^{\prime}(X-x)$. Obviously, $T^{\prime}=0$ when $x=\frac{1}{2} X$. Since $f^{\prime \prime}(x) \leq 0$ for a concave down function, $T^{\prime}>0$ when $x<\frac{1}{2} X$ and $T^{\prime}<0$ when $x>\frac{1}{2} X$. Hence, $T$ is maximum when $x_{1}=x_{2}=\frac{1}{2} X$.

\section{Induction step:}

We split $\sum_{i=1}^{n} f\left(x_{i}\right)$ into the first $n-1$ elements and $x_{n}$. We already know that for the first $n-1$ elements, the sum $\sum_{i=1}^{n-1} f\left(x_{i}\right)$ is maximum when $x_{1}=x_{2}=\cdots=x_{n-1}$. Therefore, after choosing the best $x_{n}$, the remaining $X-x_{n}$ should be equally distributed to the $n-1$ elements.

Hence, the sum $\sum_{i=1}^{n} f\left(x_{i}\right)$ is maximum for the value of $x_{n}$ that maximizes $T(x)=f(x)+(n-1) f\left(\frac{X-x}{n-1}\right)$. By repeating similar analysis as for the base case, we get

$$
\begin{aligned}
T^{\prime}(x)=f^{\prime}(x)-f^{\prime}\left(\frac{X-x}{n-1}\right) & \geq 0 \text { if } x<\frac{1}{n} X \\
& =0 \text { if } x=\frac{1}{n} X \\
& \leq 0 \text { if } x>\frac{1}{n} X
\end{aligned}
$$

That is, the sum is maximum when $x_{i}=X / n$.

\footnotetext{
${ }^{6}$ In this discussion, we define as $f^{\prime \prime} \leq 0$.
} 